\title{
Satellite Formation during Coalescence of Unequal Size Drops
}

\author{
F. H. Zhang, ${ }^{1}$ E. Q. Li, ${ }^{1}$ and S. T. Thoroddsen ${ }^{1,2}$ \\ ${ }^{1}$ Mechanical Engineering, National University of Singapore, 9 Engineering Drive 1, Singapore 117576 \\ ${ }^{2}$ King Abdullah University of Science and Technology, Thuwal, Saudi Arabia.
}

(Received 19 November 2008; published 12 March 2009)

\begin{abstract}
The coalescence of a drop with a flat liquid surface pinches off a satellite from its top, in the well-known coalescence cascade, whereas the coalescence of two equally sized drops does not appear to leave such a satellite. Herein we perform experiments to identify the critical diameter ratio of two drops, above which a satellite is produced during their coalescence. We find that the critical parent ratio is as small as 1.55 , but grows monotonically with the Ohnesorge number. The daughter size is typically about $50 \%$ of the mother drop. However, we have identified novel pinch-off dynamics close to the critical size ratio, where the satellite does not fully separate, but rather goes directly into a second stage of the coalescence cascade, thus generating a much smaller satellite droplet.
\end{abstract}

DOI: 10.1103/PhysRevLett.102.104502

PACS numbers: 47.55.df, 47.35.Pq

The partial coalescence of drops is a fascinating process involving topological changes, with both coalescence and pinch-off singularities [1-7]. The resulting drop-size distribution is of direct importance in a wide variety of twophase flow phenomena, such as rain formation in clouds [8], the coarsening of emulsions [9], and the effectiveness of industrial sprays [10]. Partial coalescence is also an intriguing natural way of generating very small droplets without external input of energy. This satellite production has been widely studied for a drop at a flat surface but is still not fully understood. One of the remaining questions is under which conditions does partial coalescence occur for two drops of different sizes and what is the size of the resulting satellite?

In sprays and other two-phase flow, drops are brought into contact by the random relative motions, often in a turbulent bulk flow field, making direct observation difficult. Figure 1 shows our setup used to systematically study the coalescence of two differently sized drops. The key to these experiments is to deposit a small droplet onto a larger hemispherical drop, resting on a steel nozzle, while limiting the approach velocity, and deformation of the drops as they start to coalesce. This is done using gravity-driven pinch-off from minute glass nozzles $(d \sim 10 \mu \mathrm{m})$, which have been sprayed with hydrophobic paint. The nozzles were made by melting a capillary tube in a glass puller. Following the release, the drop usually rebounds once or twice on the hemisphere, before the onset of coalescence, thereby having very small relative velocity. Counterintuitively, the onset of coalesce frequently occurs as the drops are moving apart, as has been elucidated by Manica et al. [11,12]. To retain nearly spherical shapes, the released droplets were kept small, i.e., between 0.45 to $1.52 \mathrm{~mm}$ in diameter. This is significantly smaller than the capillary length $a=\sqrt{\sigma / \rho g}$, where $g$ is acceleration due to gravity, $\sigma$ is the surface tension, and $\rho$ the density of the liquid. The liquids used here are distilled water and glycerin/water mixtures, whose capillary lengths are $2.7 \mathrm{~mm}$ and about $2.4 \mathrm{~mm}$, respectively. The corresponding Bond numbers, $\mathrm{Bo}=\rho g R_{m}^{2} / \sigma$ are always less than 0.1. $R_{m}$ is the radius of the mother drop. Gravity therefore has no significant effect $[3,6]$.

The idealized problem has no external velocity, with the two drops stationary at the onset of the coalescence. The motions which are driven by the release of free-surface energy are therefore characterized by the capillary-inertial velocity $v_{\sigma}=\sqrt{\sigma / \rho R_{m}}$, suggesting evolution at a constant Weber number $\mathrm{We}=\rho R_{m} v_{\sigma}^{2} / \sigma=1$. The influence of viscosity on the dynamics is correspondingly described in terms of the Ohnesorge number

$$
\mathrm{Oh}_{m}=\mu / \sqrt{\rho \sigma R_{m}},
$$

where $\mu$ is the liquid dynamic viscosity.

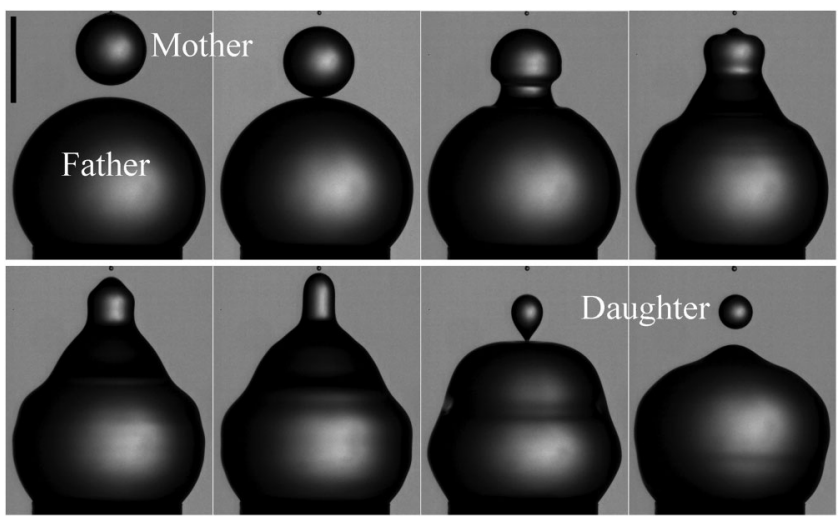

FIG. 1. Typical sequence for a satellite pinch-off, for distilled water drops with $D_{f} / D_{m}=2.72$. The very fine glass tip is visible at the top edge of the images. The satellite is 0.48 times the size of the mother drop. Coalescence starts in the second panel and subsequent images are shown 0.27, 0.67, 0.93, 1.2, 1.8 and $4.6 \mathrm{~ms}$ later. The scale bar is $1 \mathrm{~mm}$ long. 


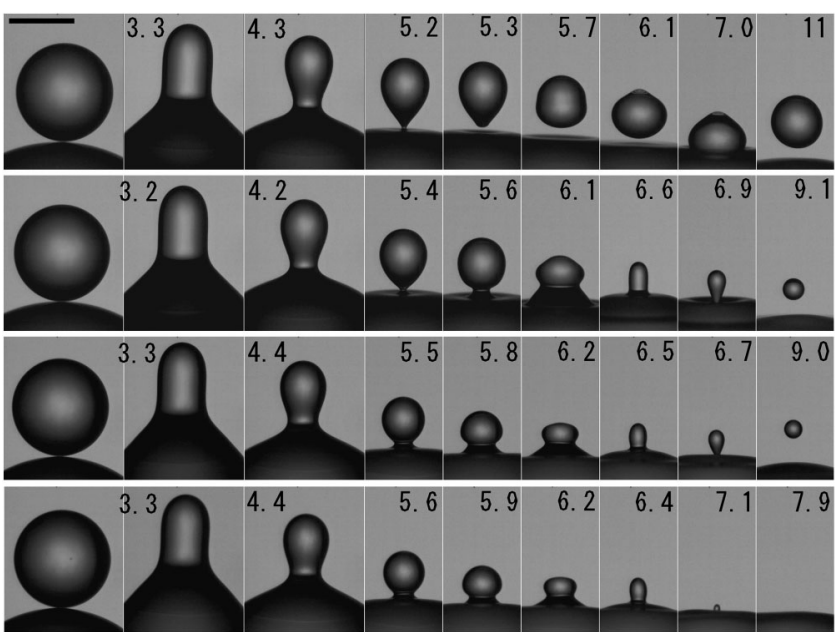

FIG. 2. The coalescence shapes for drops of $30 \%$ glycerin by volume $\left(\mathrm{Oh}_{m}=0.0116\right)$ with different size ratios, showing firststage pinch-off (top row), second-stage (two middle rows) and no pinch-off (bottom row). The parent diameter ratios from top to bottom are $D_{f} / D_{m}=2.89,2.85,2.56 \& 2.50$. Listed times are in $\mathrm{ms}$ after onset of coalescence. Scale bar is $1 \mathrm{~mm}$. See also online videos [13].

To capture the very rapid coalescence motions, we use a high-speed video camera at frame rates up to $60000 \mathrm{fps}$ (Photron SA-1). We use backlighting on a diffuser and a long-distance microscope (Leica Z-16 APO zoom).

Figure 1 shows the typical coalescence sequence, from the release of the mother drop from the nozzle to the pinchoff of the daughter drop. A prominent capillary wave is produced by the growing neck and travels up the mother drop. The wave converges at the top forming a cylindrical protrusion, which rises above the original surface and is sometimes pinched off to form a satellite. Note that the capillary wave which travels down the larger bottom hemisphere has not reached the bottom nozzle when the satellite pinches off. Reflected waves, therefore, do not affect the pinch-off dynamics.
To find the critical parent size ratio above which a satellite is pinched off, we perform a set of experiments where we slowly vary the father and mother sizes. In practice, for a given liquid, the mother drop size and $\mathrm{Oh}_{m}$ is constant for each glass nozzle, while $D_{f}$ is gradually varied, to find the region where a satellite is produced.

Figure 2 and the accompanying videos [13] show four typical coalescence sequences with slightly different parent size ratios, which lead to qualitatively different outcomes. In one case (top row) a daughter drop, about $50 \%$ of the size of the mother drop, is pinched off in essentially the same way as for the coalescence cascade at a flat surface [2]. In the two middle rows, a novel pinch-off sequence is discovered. Here, a thin neck is also produced, but the satellite does not pinch-off. Rather the neck stops shrinking and starts growing again, sending a capillary wave up the still-attached daughter. This corresponds essentially to a direct transition into the second step in the coalescence cascade [2], leading to new neck formation, which now pinches off to form a much smaller daughter droplet, with $D_{d}$ about $20 \%$ of $D_{m}$. We refer to this as a second-stage pinch-off. Finally, for a slightly smaller father drop (bottom row) a third-stage is observed to begin, but no pinchoff occurs. Figure 3(a) shows the range of parent size ratios where these three different pinch-off results are observed, for a fixed value of $\mathrm{Oh}_{m}$. The data show clearly a narrow but significant range, between the regions of first-stage pinch-off and no pinch-off, where the second-stage pinch-off occurs in a repeatable fashion.

Similar sets of experiments were carried out for many different values of $\mathrm{Oh}_{m}$, as collected in the phase diagram in Fig. 3(b). The critical parent size ratio increases monotonically with $\mathrm{Oh}_{m}$ and appears to asymptote to the results from a drop coalescing with a flat liquid surface $\left(\mathrm{Oh}_{\mathrm{cr}} \simeq\right.$ 0.026 ) [3], represented by $D_{f} / D_{m} \rightarrow \infty$. Our present, very limited data for a flat surface, indicate the critical value to be between 0.023-0.026 (broken vertical line).

On the other hand, as $\mathrm{Oh}_{m} \rightarrow 0$, the critical ratio approaches a value between 1 and 2. Experimentally, there is
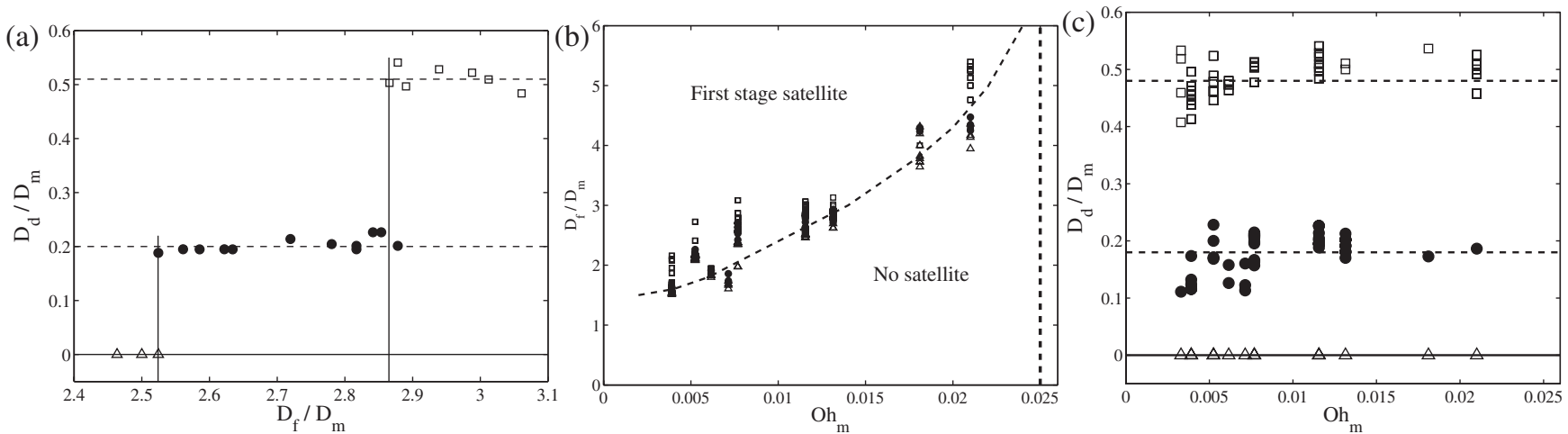

FIG. 3. The different pinch-off regimes and satellite sizes. The symbols indicate first-stage satellite ( $\square$ ), second-stage satellite (filled circles) and no satellite $(\triangle)$. (a) The size ratio of the satellite daughter vs mother droplets $D_{d} / D_{m}$, vs parent size ratio, corresponding to the same conditions as in Fig. 2. (b) The critical ratio of father and mother droplets $D_{f} / D_{m}$ vs $\mathrm{Oh}_{m}$. (c) The size ratio of the satellite daughter vs mother droplets $D_{d} / D_{m}$ vs $\mathrm{Oh}_{m}$. 
a limit to how small $\mathrm{Oh}_{m}$ can be made. As Eq. (1) shows, this can only be accomplished by reducing the liquid viscosity, or by increasing the size of the mother drop. The latter runs up against excessive deformations due to gravity, when the drop size approaches the capillary length. Using water, the smallest $\mathrm{Oh}_{m} \sim 0.0039$, before deformation starts affecting the results. Within these constraints, the smallest size ratio of the parent droplets, which we observed to pinch-off a satellite, is 1.55 .

Figure 3(c) shows the normalized size of the satellites, $D_{d} / D_{m}$, for the data in Fig. 3(b). There is a fairly large variability in this ratio but the average value, for each stage, is fairly independent of the $\mathrm{Oh}_{m}$ and the parent size ratio. The spread in the data is most likely attributable to small relative velocities between the drops when the coalescence starts, as was demonstrated in Ref. [14] for coalescing bubbles. For the first-stage pinch-off the daughter diameter is $48 \%$ of the mother diameter and for the second-stage pinch-off this ratio is $18 \%$, slightly less than $0.48^{2}=0.23$, as expected for a perfect second stage.

Figure 4(a) shows the time duration from onset of coalescence until the pinch-off of the satellite [2], for a fixed $\mathrm{Oh}_{m}=0.0116$ as in Fig. 3(a). This time is normalized by the capillary time $\tau=\sqrt{\rho R_{m}^{3} / \sigma}$. For the first-stage pinchoff this normalized time decreases with increasing parent ratio $D_{f} / D_{m}$, as is indicated by the arrow. The secondstage pinch-off obviously takes a longer time, but there is no apparent increase when the parent ratio decreases, as might be expected. This may be due to different minimum neck shapes, see Fig. 5. Figure 4(b) shows this dimensionless time over a range of $\mathrm{Oh}_{m}$. At the critical boundary between first and second-stage pinch-offs, this time is approximately a constant $t / \tau \simeq 2.1$ (solid line). This value is significantly larger than for a drop coalescing with a flat surface, where $t / \tau \sim 1.0-1.5$ [14]. The time for a secondstage pinch-off can be estimated by multiplying the firststage pinch-off time by the factor $\left[1+\left(D_{d} / D_{m}\right)^{1.5}\right]$, where we have added the first- and second-stage durations using the above scaling relation between time and diameter, providing the horizontal broken line in Fig. 4(b).

Whether the coalescence is partial or full is determined by the competition between the vertical and horizontal rates of collapse $[3,7]$. The two main factors influencing partial coalescence are the capillary waves and drainage flow from mother to father drops. Our results give new insight into the dynamics which determine the pinch-off boundary. Capillary waves not only carry momentum upwards lifting the top of the drop, but also impart inertia towards the center of the neck, as the wave travels down. Viscosity not only dissipates the capillary waves, but also reduces the drainage rate. This drainage is driven by the overall difference in capillary pressure between the two drops $\Delta p=4 \sigma\left(D_{m}^{-1}-D_{f}^{-1}\right)$, which will be enhanced by a larger parent ratio. This explains why the larger parent ratio gives a larger critical $\mathrm{Oh}_{m}$, in Fig. 3(b), as the larger pressure difference can overcome larger viscous stresses.
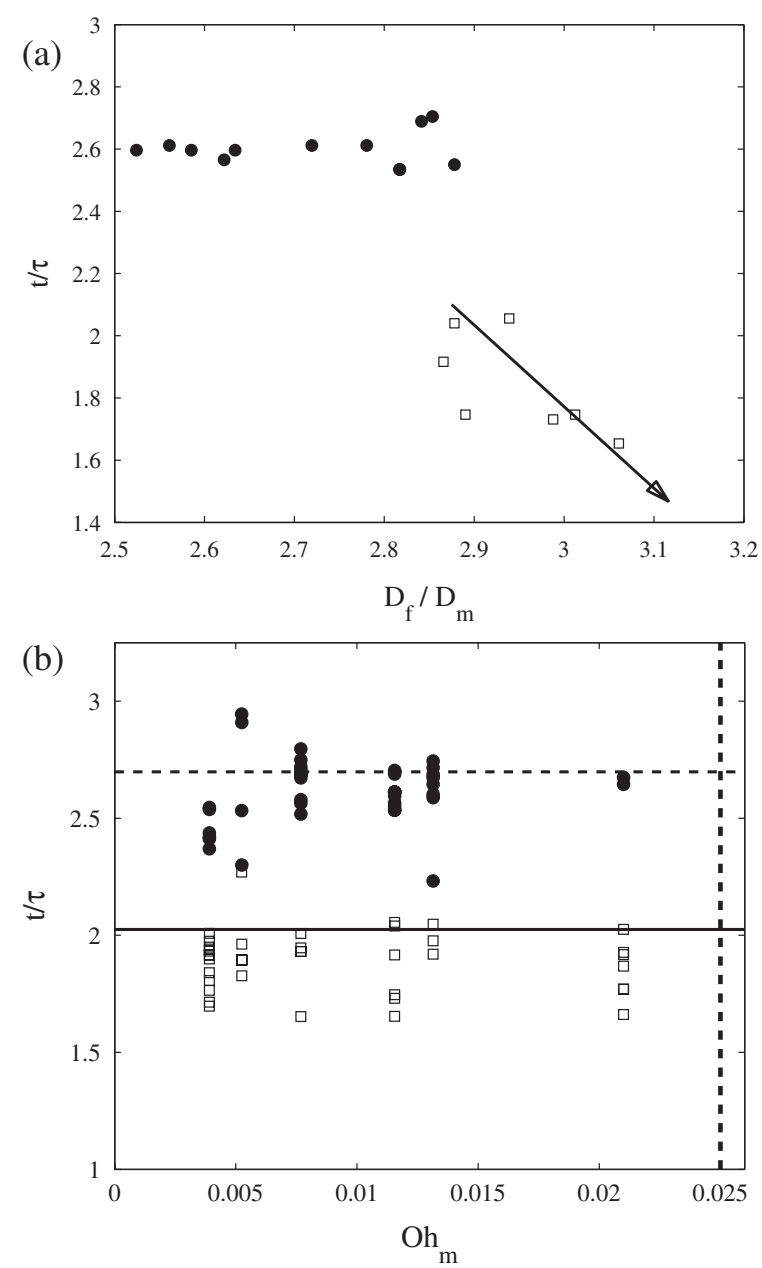

FIG. 4. The time duration from start of coalescence to satellite pinch-off, normalized by the capillary time $\tau$. For first-stage ( $\square$ ) and second-stage pinch-off (filled circles). (a) Data corresponding to Fig. 3(a). (b) Time vs $\mathrm{Oh}_{m}$ over a wide range of parent size ratios $D_{f} / D_{m}$.

The above arguments address the boundary from firststage pinch-off to no pinch-off, but do not predict the presence of the second-stage pinch-off, where the local configuration at the neck plays an important role. Figure 5 shows close-up images of the neck shapes during the contrasting pinch-off scenarios. In Fig. 5(b) the neck comes very close to pinching off, with a minimum diameter $5 \%$ of $D_{m}$. However, the axial curvature $\kappa$ is significantly sharper than the azimuthal curvature $1 / R_{\min }$, which is also drawn in the figure. Therefore the net capillary force, $\Delta p \sim \sigma\left(\kappa-R_{\min }^{-1}\right)$, tends to expand the neck, not squeeze it towards pinch-off. Furthermore, for the firststage pinch-off case, shown in Fig. 5(a), the two curvatures are essentially equal, suggesting no capillary driving force. Fluid inertia and Bernoulli pressure therefore drive the pinch-off to the final self-similar stage [15]. Note that the father drop is essentially flat when the pinch-off or near pinch-off occurs (Fig. 2), akin to the slightly reentrant shapes for a drop pinching off from a nozzle [16]. 

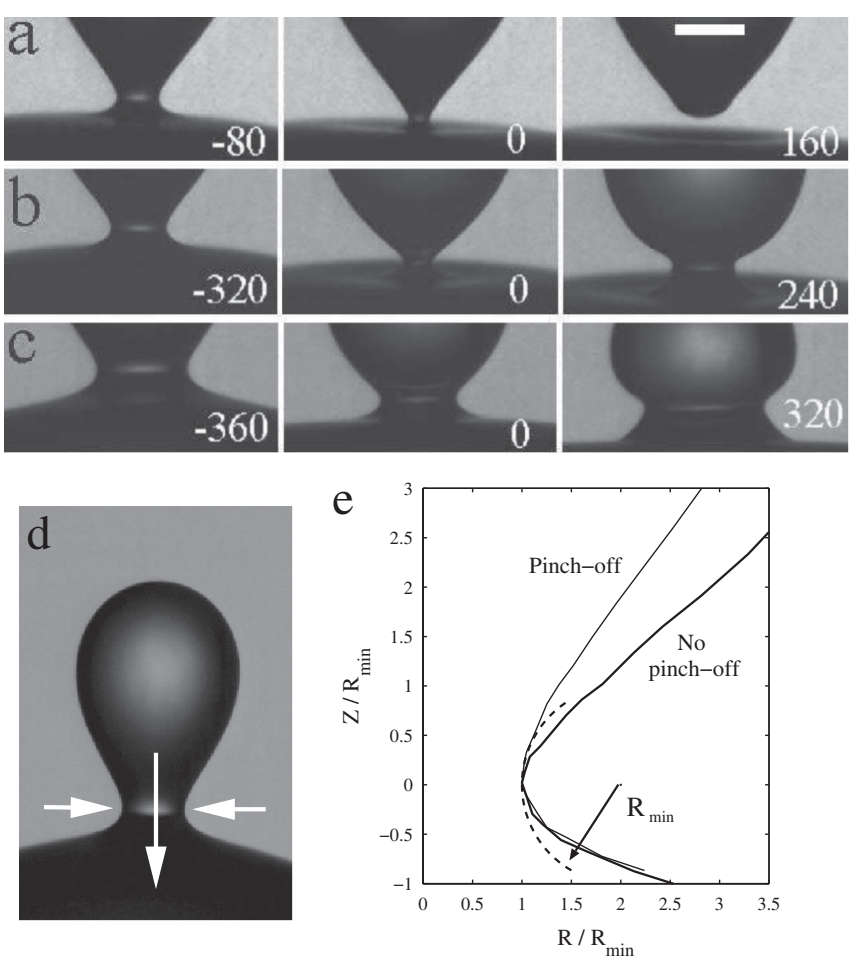

FIG. 5. Close-up of the drop shapes for first-stage (a) and second-stage pinch-offs (b),(c), for the same conditions as in Fig. 2. Scale bar is $200 \mu \mathrm{m}$ and times are in $\mu \mathrm{s}$. (d) Schematic of competing motions in the neck. (e) Neck shapes for the cases in (a) and (b). The shapes are traced when $R_{\min }=41 \mu \mathrm{m}$, which is the minimum radius observed in the sequence in (b).

The work herein has found the critical size ratio of two drops below which no daughter satellite is produced during the coalescence of two drops. The question has not been addressed experimentally before. Blanchette \& Bigioni [3] mention in passing, that the drop ratio must be larger than 2 , based on numerics. Their most recent numerical work, published after submission of our manuscript [17], refines this estimate of the size ratio and is in reasonable quantitative agreement with our experiments, but does not observe second-stage pinch-off.

The critical parent ratio reduces monotonically as $\mathrm{Oh}_{m}$ gets smaller, as in Fig. 3(b). This reduction appears to asymptote to a value of about 1.5 when $\mathrm{Oh}_{m}$ is vanishingly small, as the drop liquid is truly inviscid. This is consistent with the experiments and simulations of Menchaca-Rocha et al. [18] where no satellite is generated for the coalescence of two mercury drops of equal size, for Oh as small as $4 \times 10^{-4}$. For coalescing bubbles, however, the critical parent ratio can be as small as 0.85 [14]. Our experiments therefore indicate that the coalescence of two drops will never produce satellites from both of the drops, only from the smaller drop.

The experiments have revealed a robust second-stage pinch-off at the boundary of the pinch-off region. Similar evolution has been observed during the pinch-off of a drop (Fig. 12 in [19]) and less clearly for coalescence of polymeric drops with their bulk phase $[5,6]$. This phenomenon represents a great challenge to numerical simulations [17], when the minimum neck size must be set in an ad hoc manner. This second-stage, or a possible third-stage pinchoff (bottom Fig. 2) could therefore produce smaller satellites than previously observed.

In conclusion, we have determined the critical size ratio of two drops in air, above which a satellite is pinched off during their coalescence. This ratio is as small as 1.55 , but grows with increasing viscosity in terms of $\mathrm{Oh}_{m}$. We have also discovered a new kind of satellite generation, which occurs at the critical boundary, where two coalescence steps occur in succession, without intermediate detachment. This produces much smaller satellite droplets than during coalescence with a flat surface.

We thank Victor Shim and the Impact Mechanics Laboratory at NUS for use of their high-speed cameras.

[1] G. E. Charles and S. G. Mason, J. Colloid Sci. 15, 105 (1960).

[2] S. T. Thoroddsen and K. Takehara, Phys. Fluids 12, 1265 (2000).

[3] F. Blanchette and T.P. Bigioni, Nature Phys. 2, 254 (2006).

[4] H. Aryafar and H. P. Kavehpour, Phys. Fluids 18, 072105 (2006).

[5] X. Chen, S. Mandre, and J. J. Feng, Phys. Fluids 18, 092103 (2006).

[6] P. T. Yue, C.F. Zhou, and J. J. Feng, Phys. Fluids 18, 102102 (2006).

[7] T. Gilet et al., Phys. Rev. E 75, 036303 (2007).

[8] Y. Xue, L.-P. Wang, and W. W. Grabowski, J. Atmos. Sci. 65, 331 (2008).

[9] V.E. Ziegler and B. A. Wolf, Polymer 46, 9265 (2005).

[10] E. Villermaux, Annu. Rev. Fluid Mech. 39, 419 (2007).

[11] R. Manica et al., Langmuir 24, 1381 (2008).

[12] N. Bremond, A. R. Thiam, and J. Bibette, Phys. Rev. Lett. 100, 024501 (2008).

[13] See EPAPS Document No. E-PRLTAO-102-069912 for supplementary videos. For more information on EPAPS, see http://www.aip.org/pubservs/epaps.html.

[14] F. H. Zhang and S. T. Thoroddsen, Phys. Fluids 20, 022104 (2008).

[15] R. F. Day, E. J. Hinch, and J. R. Lister, Phys. Rev. Lett. 80, 704 (1998).

[16] J. C. Burton and P. Taborek, Phys. Rev. Lett. 101, 214502 (2008).

[17] F. Blanchette and T. P. Bigioni, J. Fluid Mech. 620, 333 (2009).

[18] A. Menchaca-Rocha, A. Martinez-Davalos, R. Nunez, S. Popinet, and S. Zaleski, Phys. Rev. E 63, 046309 (2001).

[19] S. T. Thoroddsen, T. G. Etoh, and K. Takehara, Phys. Fluids 19, 052101 (2007). 